\title{
Eclipsing binary statistics - Theory and observation
}

\author{
S. Söderhjelm and J. Dischler
}

Lund Observatory, Box 43, 22100 Lund, Sweden

e-mail: [staffan; johann] @astro.lu.se

Received 15 December 2004 / Accepted 20 May 2005

\section{ABSTRACT}

\begin{abstract}
The expected distributions of eclipse-depth versus period for eclipsing binaries of different luminosities are derived from large-scale population synthesis experiments. Using the rapid Hurley et al. BSE binary evolution code, we have evolved several hundred million binaries, starting from various simple input distributions of masses and orbit-sizes. Eclipse probabilities and predicted distributions over period and eclipsedepth $(\mathrm{P} / \Delta m)$ are given in a number of main-sequence intervals, from O-stars to brown dwarfs. The comparison between theory and Hipparcos observations shows that a standard (Duquennoy \& Mayor) input distribution of orbit-sizes $(a)$ gives reasonable numbers and $\mathrm{P} / \Delta m$-distributions, as long as the mass-ratio distribution is also close to the observed flat ones. A random pairing model, where the primary and secondary are drawn independently from the same IMF, gives more than an order of magnitude too few eclipsing binaries on the upper main sequence. For a set of eclipsing OB-systems in the LMC, the observed period-distribution is different from the theoretical one, and the input orbit distributions and/or the evolutionary environment in LMC has to be different compared with the Galaxy. A natural application of these methods are estimates of the numbers and properties of eclipsing binaries observed by large-scale surveys like Gaia.
\end{abstract}

Key words. methods: miscellaneous - binaries: general - binaries: eclipsing - stars: formation - stars: evolution stars: statistics

\section{Introduction}

Eclipsing binaries are important tools for investigating stellar parameters, but with periods and eclipse-depths taken as provided by Nature. Some pioneering statistics for the perioddistributions for eclipsing binaries of different types were given by Farinella \& Paolicchi (1978) and Antonello et al. (1980), but the distribution of the eclipse-depths has less often been studied in its own right. An important reason is that such statistics are very incomplete. Eclipsing binaries have been discovered from non-systematic searches, and their physical natures are too diverse for meaningful statistics. Forthcoming large-scale surveys like Gaia will however observe large numbers of eclipsing binaries, and it is clearly interesting to be able to give at least crude estimates of the numbers expected in different parts of the HR diagram.

The two most important observable parameters for an eclipsing binary are the period and the (maximum) eclipse depth. We also generally know at least an approximate absolute magnitude or mass for the system. For all-sky surveys like Hipparcos or Gaia, we have an "all-age/all-mass" sample, and as a first theoretical study, it seems reasonable to evolve timeand space-invariable initial distributions of masses and orbits during a 12 Gyr evolution of a Galactic disk. We will thus use a large sample of binaries, trying to reproduce their present characteristics by a large-scale population synthesis. The eclipse probability depends mainly on the ratio between the stellar radii and the orbit size, and we get appreciable numbers of eclipsing binaries only at periods of weeks or days. Because of orbital evolution, however, such a short-period system may have started out in a much wider orbit, and to keep this possibility, the population synthesis is started with a very wide distribution of orbit-sizes $(a)$, and typically millions of original binaries are needed to get a few hundred eclipsing pairs.

For all the interesting (short) periods, the evolution includes episodes of mass-transfer or a common-envelope phase, and the parameters of the final binaries cannot be obtained without following this evolution in detail. The rapid binary evolution code by Hurley et al. (2002), hereafter BSE, is here a fundamental tool. Using large numbers of analytic interpolation formulae, the BSE code summarizes and extends data from a large number of detailed evolution models in a unified structure where a given binary with arbitrary mass, metallicity and orbit can be followed in time. The rapid results obtainable with BSE enables the large-scale studies needed to get sufficient numbers of eclipsing pairs, but the realism of the final data also depends crucially on the accuracy and parameter choices in the BSE code.

The basic assumptions and parameters used in our theoretical investigations are described in Sects. 2 and 3. Because the eclipse probabilities vary mostly with the stellar radii, it is useful to combine data in smaller parts of the HR diagram, 
where the radii are fairly similar. This binning along the main sequence is described in Sect. 4, and in Sect. 5 we present some typical results. Section 6 explores the sensitivity of these results to various changes of the input assumptions, before the theory is compared with observed data in Sects. 7 (Hipparcos) and 8 (LMC). Section 9 gives our preliminary conclusions, and the Appendix shows some sample tables.

\section{Population synthesis for binaries}

We try to reproduce galactic disk data starting from simple original distributions of masses and orbits. We simply assume a constant Star Formation Rate (0-12 Gyr), a timeindependent IMF (Kroupa 2001), with

$\xi(m) \propto m^{-\alpha_{i}}$,

and with

$\alpha_{0}=0.3, \quad 0.03<m / M_{\odot}<0.08$

$\alpha_{1}=1.8, \quad 0.08<m / M_{\odot}<0.50$

$\alpha_{2}=2.7, \quad 0.50<m / M_{\odot}<1.00$

$\alpha_{3}=2.3, \quad 1.00<m / M_{\odot}<50.0$.

The metallicity distribution in the synthesis program is ageindependent and uniform in $[\mathrm{Fe} / \mathrm{H}]$, and several runs with different upper and lower limits are combined in order to mimic the observed distributions. For the galactic samples, $10 \%$ of the $[\mathrm{Fe} / \mathrm{H}]$-values are in the interval $[0.1,0.23], 70 \%$ in the interval $[-0.3,0.1]$ and $20 \%$ in the interval $[-0.8,-0.3]$. This gives a total distribution as broad as e.g. the one shown in Nordström et al. (2004).

To allow later scalings to different multiplicity fractions, we either make all the IMF mass fragments into single stars, or all to binaries. The binary runs are of most interest to us, and we have to assume a distribution $f(a, q, e)$ of orbit-sizes, initial mass-ratios and initial eccentricities. Even if it is clear that this is in general not separable, we make the simplistic first assumption $f(a, q, e)=f(a) \times f(q) \times f(e)$.

The $q$-distribution (with $q<1$ for the ratio of the originally smaller to the larger mass) is mostly the rather ad hoc compromise function

$f(q)=0.446 \times[1+2 n(q-0.2,0.3)+2 n(q-1.0,0.05)]$

with $n(m, s)=\exp \left(-m^{2} /\left(2 s^{2}\right)\right)$. The narrow peak at $q=1$ is reduced as compared with Söderhjelm (2000), because it is not seen by e.g. Mazeh et al. (2003), or as a much broader feature by Halbwachs et al. (2003). Its total contribution to the above $f(q)$ is only about $6 \%$, and it is relatively unimportant in the present study. For comparison, we have used also the gaussian distribution given by Duquennoy \& Mayor (1991), as well as a random pairing scheme, each component of the binary picked independently from the IMF and paired randomly. See Sect. 6.

The distribution of the semi-major axes of the binaries is usually taken as

$$
f(\lg a)=0.269 \times n(\lg a-1.5,1.5) \quad-3<\lg a[\mathrm{au}]<5
$$

which is close to the (period-)relation given by Duquennoy \& Mayor (1991). Some other choices are tested in Sect. 6.

The eccentricities are distributed thermally for the largest orbits,

$$
f(e) \propto 2 e \quad a>1000 \mathrm{au}
$$

turning smoothly into a uniform distribution at $a<10$ au. The tidal evolution built into the BSE-code usually produces a rapid circularization for periods below about 10 days.

The BSE routines include a number of (sometimes poorly known) parameters, but to keep the amount of computations reasonable, we used only the default values given in Table 3 in Hurley et al. (2002). If these parameters can be trusted, the BSE code gives for each input binary the actual masses, temperature and luminosities of the components, as well as the orbit size and shape, even when the evolution includes episodes of mass-transfer and common-envelope evolution. A minor problem occurs at brown dwarf masses, since the BSE models are accurate only down to a bona fide stellar mass-limit around $0.08 M_{\odot}$. For any component of lower mass, we added an extra grid with data due to Baraffe et al. (1998), giving more realistic cooling brown dwarfs.

For orbits with periods above about 5 years, each component in the binary evolves independently, and we can make a binary from two single-star models, using analytical approximations to the single-star evolutionary tracks given by Hurley et al. (2000), and again with the Baraffe brown dwarf extension. As a check of the importance of the full BSE models, we have used in parallel this $\mathrm{S}+\mathrm{S}$ approximation. There is the non-trivial problem of how to evolve the orbit (size and eccentricity) when one or both of the components lose mass. We used here a simplistic prescription with the semimajor axis inversely proportional to the total remaining mass in the system, and with a circularization time-scale given by $\lg t_{\text {circ }}[\mathrm{yr}]=23+10 \lg a[\mathrm{au}]-8 \lg R\left[R_{\odot}\right]$ (where $R$ is the radius of the largest star in the system). When any of the components reached its Roche-lobe, the system was removed. Still, this $\mathrm{S}+\mathrm{S}$ approach gives a qualitatively similar (post-evolution) a-distribution, with a hump before an obvious contact-limit. Figure 1 shows the short-period end of the a-distributions for the FG-bin (see Sect. 4), as calculated by BSE and by the $\mathrm{S}+\mathrm{S}$ approximation. In this and other cases, the $\mathrm{S}+\mathrm{S}$ model gives (counter-intuitively) more short-period pairs than the more realistic BSE calculations.

Both for the BSE and $\mathrm{S}+\mathrm{S}$ runs, there is the standard problem of going from theoretical $\left(M_{\mathrm{bol}}, T_{\mathrm{eff}}\right)$ to observable $\left(M_{V}\right.$, $V-I$ ) coordinates, and again only illustrative transformations are used, based on the data in Flower (1996) but with misprint corrections and extensions by P. Nurmi (private comm.) and S. Söderhjelm. For cool stars, these transformations are admittedly rather poor, but their influence on the $\Delta m$-statistics is very indirect, and more elaborate models are not called for in this first study.

\section{Eclipse statistics}

The population synthesis gives a number of binaries with known properties, among them the orbit size and period and the 


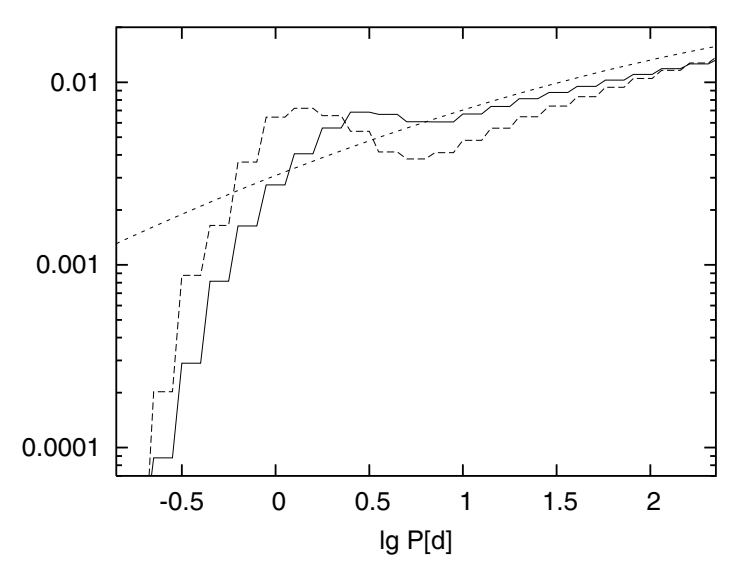

Fig. 1. The short-period part of the period-distribution for systems with F- or G-star primaries. The full line shows the BSE calculation, the dashed one the $\mathrm{S}+\mathrm{S}$ approximation, and the dotted line the smooth input distribution.

stellar radii and luminosities. The radii relative to the orbit-size $r_{\mathrm{s}}=R_{\mathrm{s}} / a$ and $r_{\mathrm{g}}=R_{\mathrm{g}} / a$ (where $\mathrm{s}$ and $\mathrm{g}$ stand for smaller and greater) are the interesting parameters, together with the relative ( $V$-band) luminosities $L_{\mathrm{s}}$ and $L_{\mathrm{g}}$, normalized to $L_{\mathrm{s}}+L_{\mathrm{g}}=1$. It is easy to show that the probability of an eclipse of any depth is simply $P_{\mathrm{e}_{0}}=r_{\mathrm{s}}+r_{\mathrm{g}}$, but we want to study the probability of an eclipse of any specified (magnitude) depth. In this first study, we have neglected all light-curve complications due to tidal deformation and/or reflection effects, and in the same vein, we also assume zero limb-darkening.

For this simple case, the light-curve can be calculated from the radii, luminosities and orbital inclination, as shown e.g. by Irwin (1962). Although the maximum (primary) eclipse depth $\Delta m$ is a well-defined function of the inclination $i$, iteration is needed for the inverse problem of deriving the function $i(\Delta m)$. With a random distribution of inclinations, the probability $P_{\mathrm{e}}(\Delta m)$ of a primary eclipse deeper than $\Delta m$ is now simply $\cos (i(\Delta m))$. As expected, the inclination at grazing eclipse $(\Delta m=\epsilon \approx 0)$ is then $i(\epsilon)=\arccos \left(r_{\mathrm{s}}+r_{\mathrm{g}}\right)$, giving $P_{\mathrm{e}}(\epsilon)=P_{\mathrm{e}_{0}}$.

For main sequence stars of roughly constant size, the $P_{\mathrm{e}}$-values decrease as $1 / a$, or as $P^{-2 / 3}$. In practice, however, a longer period also means narrower (easily missed) eclipses, and the observed bias towards smaller periods is more pronounced. To account for this effect, a nonzero $P_{\mathrm{e}}$-value is only calculated if the $\Delta m$ is larger than $0.1 \mathrm{mag}$ for at least $5 \%$ of the orbit. As shown e.g. in Söderhjelm (2005), such discovery criteria have a large impact on the derived results. In the present study, we have used the 5\%/0.1 mag limits throughout, except for a comparison in Sect. 7.

Mean values for these eclipse probabilities are meaningful only for roughly similar systems, meaning roughly similar radii, luminosities and orbit sizes. In practice, we have divided the HR diagram in a number of bins of absolute magnitude and color, and for the stars in each bin, we then further subdivide according to orbital period and $\Delta m$. The final results are histograms giving the mean $P_{\mathrm{e}}(\Delta m, P)$ in each separate HR-diagram bin. These eclipse probabilities are interesting in themselves, and one may compare e.g. the BSE and $\mathrm{S}+\mathrm{S}$ results.
However, for comparisons with observed eclipsing binaries, another type of probability is more interesting, namely the fraction of eclipsing binaries relative to all stars (binary and single) in the same HR diagram bin. In order to derive such a normalized probability $O_{\mathrm{e}}(\Delta m, P)$, we multiply the corresponding $P_{\mathrm{e}}(\Delta m, P)$ by the number of binaries in that particular period range and divide by the total number of binaries (of all periods) and single stars in the specified HR diagram bin. The $O_{\mathrm{e}}$ values are sensitive to the period-distribution of the binaries, and also to the absolute frequency of double relative to single stars. In our model, we assume a nominal $80 \%$ duplicity fraction, in the sense that only $20 \%$ of the mass fragments from the IMF are kept as single stars, while $80 \%$ are split into doubles. Because a single has higher mass than the primary in a double created from the same mass fragment, the duplicity fraction when comparing singles and primaries of the same mass is lower (and variable along the main sequence).

\section{Covering the MS}

The overall $M_{V} /(V-I)$ color-magnitude diagram for all metallicities and all ages shows a very wide main sequence, and the binning selected is similarly coarse. Figure 2 shows the separation into 22 regions, together with some typical isochrones. The color-boundaries are designed for a basic separation into MS, giant and wd parts, and on the MS, the $M_{V}$-intervals can be (illustratively) translated to spectral type. The naming-convention is shown in Fig. 2 and Table 1.

The simplistic approach of making a single run with the full IMF and the full 0-12 Gyr age range would require an inconvenient number of simulated stars, and would still give in the end very few upper MS eclipsing binaries. Instead, we have made a series of partial runs, each covering mass-sums from a lower limit to the BSE maximum (around $45 M_{\odot}$ ), and with a maximum age that allows the lowest-mass pairs to evolve past the MS stage. In this way, most of the objects at any time populating this MS-bin can be generated (allowing for massive binaries evolving downwards, but with no expectation that lower-mass pairs may ever become brighter than a single star with their combined mass). For the upper MS, one or two of these partial runs were designed to populate each bin, and in order to have large samples of close binaries, the total numbers (of wider systems) were very large. As described in Sect. 2, each "run" was also routinely split in three or more partial ones with different ranges of metallicity)

For example, the $\mathrm{O}$ bin was created from a total of 19 million systems with mass-sums larger than $16 M_{\odot}$ and ages 0-12 Myr. For the A bin, 23 million simulated systems with mass-sums above $1.35 M_{\odot}$ were used, with ages 0-3000 Myr. In total, we spent about 2500 (2 Ghz Pentium 4) CPU-hours to simulate about 250 million binaries. The magnitude limits defining the different bins are given in Table 1. Because of the IMF slope, the median absolute magnitude in a bin is not central between these bin-limits, but shifted towards the faint edge, or, for the least massive dwarfs, towards the bright edge instead. 


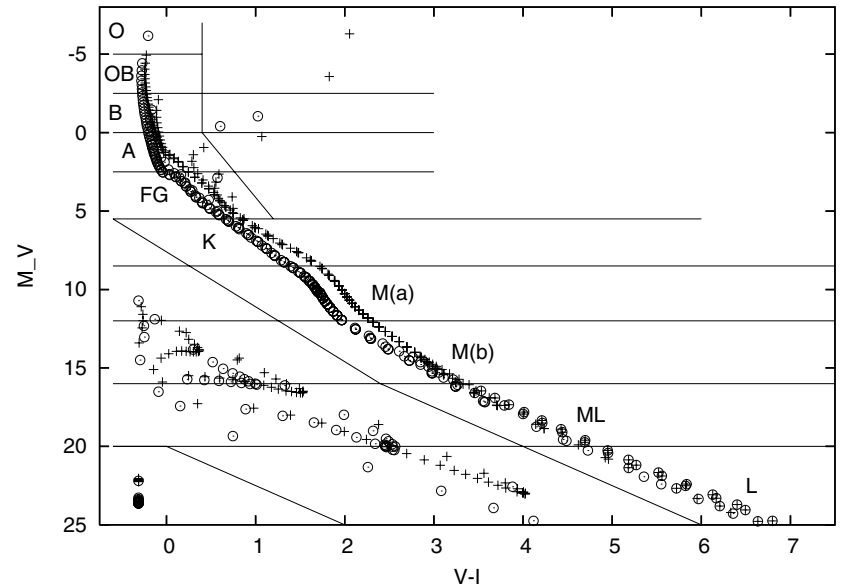

Fig. 2. The binning in the CM-diagram, with the main-sequence bins labelled with approximate spectral types. About 100 stellar models $\left(0.03-45 M_{\odot}\right)$ are plotted at age 10, 100, 1000, 3000 and $10000 \mathrm{Myr}$, for $[\mathrm{Fe} / \mathrm{H}]=0$ (crosses) or $[\mathrm{Fe} / \mathrm{H}]=-2$ (open circles), showing the main locations for the MS and WD sequences.

Table 1. The magnitude limits for the main-sequence bins, and the median absolute magnitude for the stars in the bin, for the standard input assumptions.

\begin{tabular}{lcr}
\hline \hline Bin-id & $M_{V}$-interval & $\left\langle M_{V}\right\rangle$ \\
\hline O & $<-5$ & -5.31 \\
OB & $-5.0--2.5$ & -3.04 \\
B & $-2.5-0.0$ & -0.55 \\
A & $0.0-2.5$ & 1.79 \\
FG & $2.5-5.5$ & 4.35 \\
K & $5.5-8.5$ & 7.15 \\
M(a) & $8.5-12.0$ & 10.70 \\
M(b) & $12.0-16.0$ & 13.99 \\
ML & $16.0-20.0$ & 17.71 \\
L & $20.0-25.0$ & 22.44 \\
\hline
\end{tabular}

\section{Typical results}

The basic output from our studies are histograms (with a 0.15 dex binning in $\lg P$ ) giving the mean probability of observing an eclipse of at least a (maximum) depth $\Delta m$. Figure 3 shows a plot of these data for the FG-bin, with the $0.1 \mathrm{mag}$ curve at the top and the $1.5 \mathrm{mag}$ curve at the bottom. Because the probabilities for deep eclipses are so small, a logarithmic scale is necessary to show the details. For the $O_{\mathrm{e}}$-plot in Fig. 4 , we have taken the summed $P_{\mathrm{e}}$-values in each period bin, and divided by the total number of (singles and binaries of all periods) in the whole FG-bin. (More specifically, the $O_{\mathrm{e}}$-values are defined per 0.3 dex period-interval and not the 0.15 dex histogram spacing. We have also used the $80 \%$ duplicity defined in Sect. 3.) The $O_{\mathrm{e}}$-curves decrease at short periods because the number of short-period pairs decreases (cf. Fig. 1), although each such system has a high probability of eclipses. The decrease towards larger periods is shaped also by the assumed discovery-criterion, see Sect. 7.

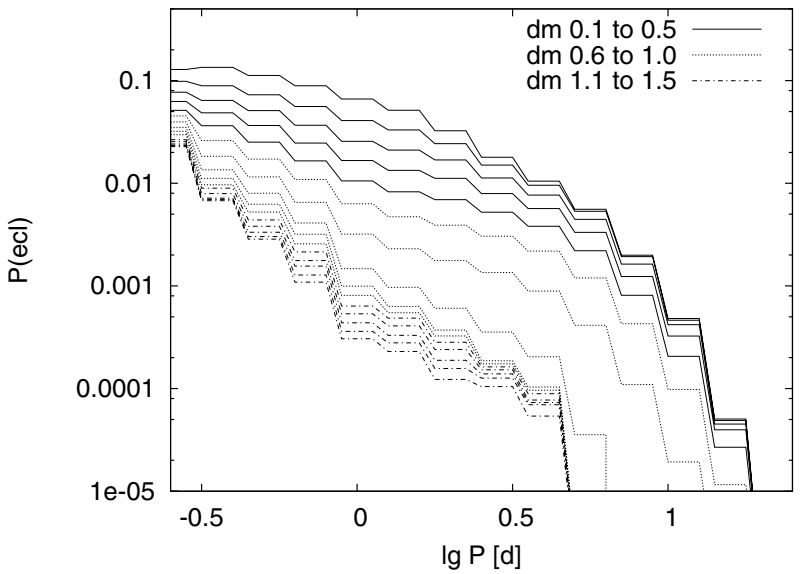

Fig. 3. The eclipse probabilities $P_{\mathrm{e}}(\Delta m, P)$ for the FG bin, as a function of the $(\log )$ period in days. Each of the lines (solid from 0.1 to $0.5 \mathrm{mag}$, dashed from 0.6 to 1.0 , and dot-dashed from 1.1 to $1.5 \mathrm{mag}$ ) corresponds to a certain maximum eclipse-depth $\Delta m$.

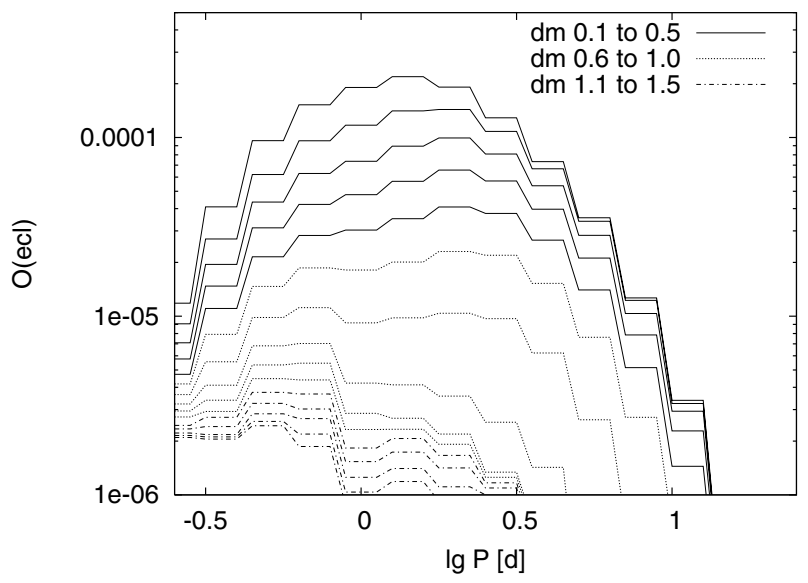

Fig. 4. The $O_{e}(\Delta m, P)$-curves, giving the fraction of eclipsing systems (per 0.3 dex in $\lg P$ ) relative to all stars, again for the FG bin. Same key for the $\Delta m$ :s as in Fig. 3.

The deeper eclipse for any eclipsing binary is due to the cooler star eclipsing the hotter. For unevolved main-sequence stars, a hotter star is always larger, and the partial primary eclipses can be shown to be never deeper than the $0.75 \mathrm{mag}$ limiting case when two equal stars mutually cover each other. A deeper primary eclipse needs a more complex evolution with Roche-lobe overflow and mass-transfer producing Algol systems with subgiant secondaries that may totally eclipse their hotter primaries.

Although typically less than $1 \%$ of the eclipsing FG systems show 1.5 mag eclipses, the ratio at the shortest periods is closer to $20 \%$, showing a much higher proportion of such Algol systems. The situation is even more complex for massive systems, as seen e.g. in Fig. 5 for the B bin. There are now characteristic humps with a high probability of deep eclipses at about 2 day and 0.4 day orbital periods. The longer-period hump is visible from A- to OB-stars, although it shifts successively towards larger periods for the larger masses. It would be interesting to investigate further which kinds of progenitor systems produce these deeply eclipsing systems, and what 


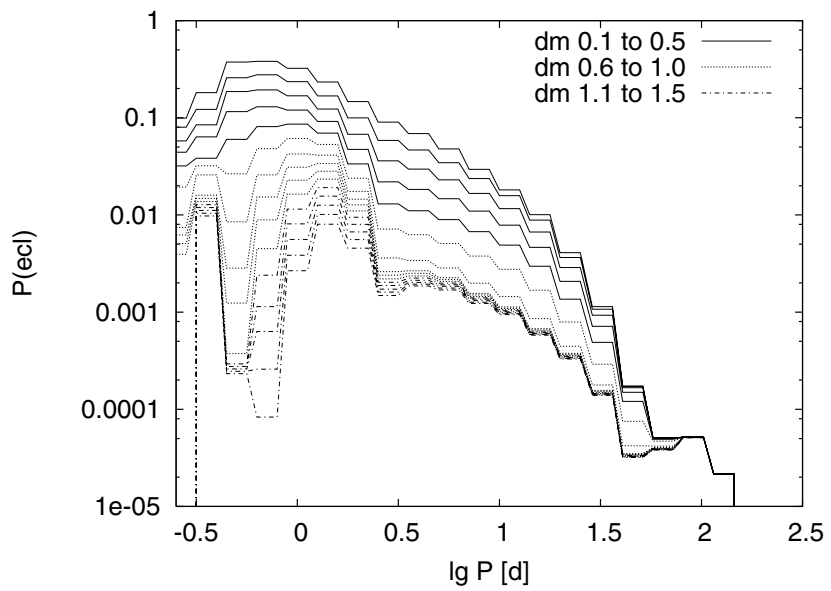

Fig. 5. The eclipse probabilities $P_{\mathrm{e}}(\Delta m, P)$ for B systems. Because more systems have undergone mass transfer, the details are more complex than in Fig. 3.

parameters influence their periods, but we have not yet tried to do so. As will be seen in Sect. 8, the hump period seems to be larger in the LMC than in the Galaxy, and more experimenting will be needed to understand all the factors involved.

Our $\mathrm{S}+\mathrm{S}$ binaries should be identical to the BSE ones as long as there is no mass-transfer between the components. In practice, there are differences already in the orbit evolution when a substantial fraction of the mass is lost from the system in the giant stage. When both stars are on the main sequence, however, the results are quite similar. The low-mass $M(\mathrm{~b})$-stars are expected to remain on the MS for more than $12 \mathrm{Gyr}$, and as seen in Fig. 6, the $\Delta m$-curves for $0.2,0.4$ and 0.6 mag are more or less equal for $\mathrm{S}+\mathrm{S}$ and BSE. In the $\mathrm{S}+\mathrm{S}$ model, the 0.8 mag curve is also almost at zero (by the "0.75-effect"), but with BSE, some originally higher-mass binaries have after a common-envelope phase actually become short-period systems with wd primaries and M-type secondaries. Going up the mainsequence, the differences between the $\mathrm{S}+\mathrm{S}$ and BSE models increase, in the sense that only BSE can create the deeply eclipsing Algol systems.

An interesting feature of bona-fide brown dwarfs is that their radii remain rather constant while they cool, making it possible to find totally eclipsing systems where the eclipsing star is much fainter than the eclipsed one. The BSE data are in this case identical to the $\mathrm{S}+\mathrm{S}$ ones, in both cases based on our implementation of the Baraffe et al. (1998) data. The $P_{\mathrm{e}}$-curves shown in Fig. 7 could be extended at a similar equidistant spacing down to quite large $\Delta m$ (but more systems would have to be simulated to get good enough statistics). The curves for 0.1 to 0.4 mag eclipse depth more or less merge, because of the improbability of close to grazing eclipses for very small stars.

In Fig. 8, we show only the largest eclipse-probabilities $(\Delta m>0.1 \mathrm{mag})$, but for several absolute magnitude bins at once. There is now a very definite trend with higher probabilities (at longer periods) for brighter stars, because they are larger. For the $\mathrm{O}$ systems, the short-period contact-limit is very apparent, with no room for shorter period systems with these large radii.

For a fuller sample of the data, see Appendix A.

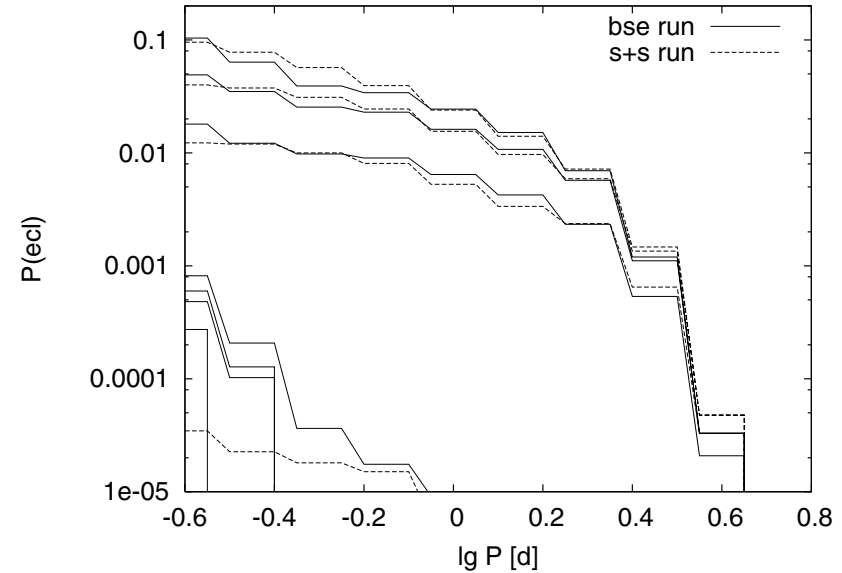

Fig. 6. Comparison of $P_{\mathrm{e}}$-data for low-mass M-stars $(M(\mathrm{~b})$ bin), as calculated with BSE (solid lines) and with a simplified single+single model (dashed lines). Values are given for (from above) $\Delta m=0.2,0.4$, $0.6, \ldots, 1.4$, but the $\mathrm{S}+\mathrm{S}$ curves for $\Delta m>0.8$ disappear below the plot area.

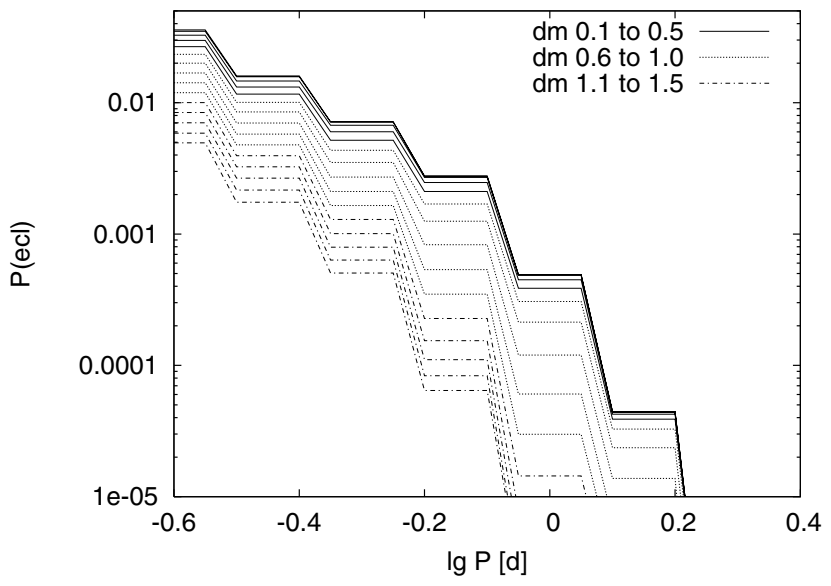

Fig. 7. The $P_{\mathrm{e}}(\Delta m, P)$-curves for brown dwarfs (ML and L bins). Standard (Fig. 3) labelling of the $\Delta m$ curves.

\section{Varying the input parameters}

All the above results are for a single set of input parameters. Even if the assumptions are reasonable, it is obviously very important to know how a different choice would affect the results. Ideally, one would like to vary one parameter at a time, redo all calculations, and see the differential change in the output. With tens of parameters to look at, the amount of computation is prohibitive, and for simplicity, we have not tried to vary the parameters in BSE. Foremost among the poorly-known ones is the notorious "common-envelope efficiency parameter", which is kept at $\alpha_{\mathrm{CE}}=3.0$. We have concentrated instead on the input distributions of orbit sizes and mass-ratios, and on the effects of the metallicity.

In order to see the differential effects of changes in $f(a)$ and $f(q)$, it is sufficient to use only a single (solar) metallicity, and also to use slightly incomplete samplings of the absolute magnitude bins. With these simplifications, we have made 5 extra sets of simulations at a number of absolute magnitude bins. Instead of the nominal a-distribution in Eq. (4), we have tried a more peaked (A1), or a more uniform (A2) one. Instead 


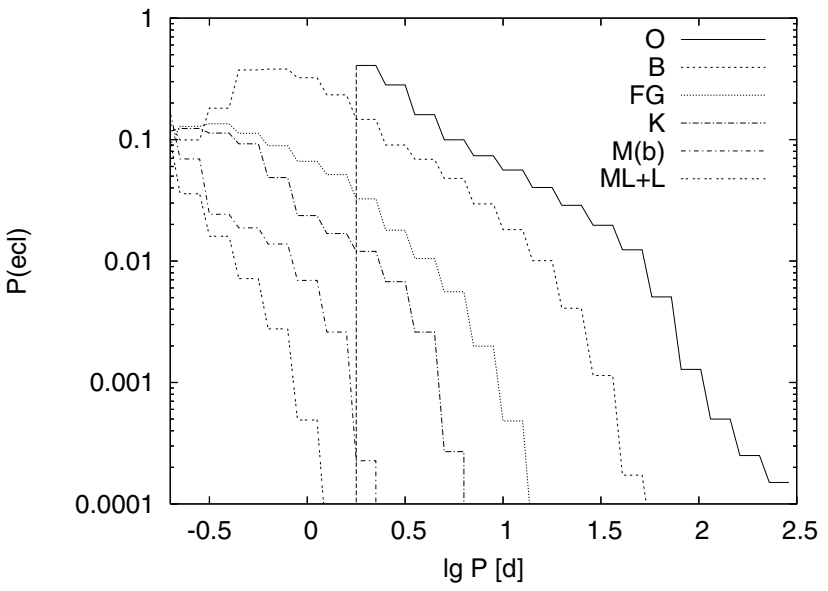

Fig. 8. The eclipse probabilities $P_{\mathrm{e}}(0.1, P)$ for a number of different MS bins, as indicated in the legend.

Table 2. The differential runs, varying the $a$ - or the $q$-distributions.

\begin{tabular}{ccc}
\hline \hline Run & $a$-distr & $q$-distr \\
\hline Std & Eq. (4) & Eq. (3) \\
A1 & $n(\lg a-1.5,1.0)$ & Eq. (3) \\
A2 & $n(\lg a-1.5,2.0)$ & Eq. (3) \\
Q1 & Eq. (4) & $n(q-0.23,0.42)$ \\
Q2 & Eq. (4) & uniform \\
Q3 & Eq. (4) & random pairing \\
\hline
\end{tabular}

of the nominal $q$-distribution in Eq. (3), we have tried either the Duquennoy \& Mayor (1991) (Q1) or a uniform (Q2) $f(q)$, neither showing any peak at $q=1$. To demonstrate the untenability of a random pairing $q$-distribution (cf. Malkov \& Zinnecker 2001), we also made a few runs (Q3) where the secondary was chosen from the same IMF as the primary, producing an $f(q)$ rising sharply towards small $q$. The assumptions are summarized in Table 2. At each of the selected MS bins, we have then four or five extra sets of $P_{\mathrm{e}} / Q_{\mathrm{e}}$-results apart from the nominal one. Because we could not make as many simulations as in the main runs, the numerical uncertainties are larger, but the uppermost $(\Delta m>0.1)$ curves are well-defined.

As expected, the $P_{\mathrm{e}}$-data are usually less affected than the $O_{\mathrm{e}}$-data, because they depend mostly on the (similar) physical characteristics of the individual components. The $O_{\mathrm{e}}$-data depend also on the close double period-distribution, which may change appreciably with the input $f(a)$ and $f(q)$. As a simple illustration, we may again look at the FG bin. Figure 9 shows the $0.1 \mathrm{mag}$ and $0.8 \mathrm{mag} O_{\mathrm{e}}$-curves for the nominal run and with the Q1-Q3 modifications. Small (Q1/Q2) changes in the $q$-distribution are seen to have a rather small impact on the $O_{\mathrm{e}}$-curves, with the flat $f(q)$ giving marginally more, and the DM one marginally fewer eclipsing binaries. As expected, however, the Q3 model gives much fewer (about a factor of 5) eclipsing binaries, even with an assumed $100 \%$ duplicity. As the primary mass increases, the predominance of small-mass secondaries gets more and more extreme, producing very few similar-mass binaries and less close binary interaction. For the OB-bin, a Q3 run gives at least 50 times fewer

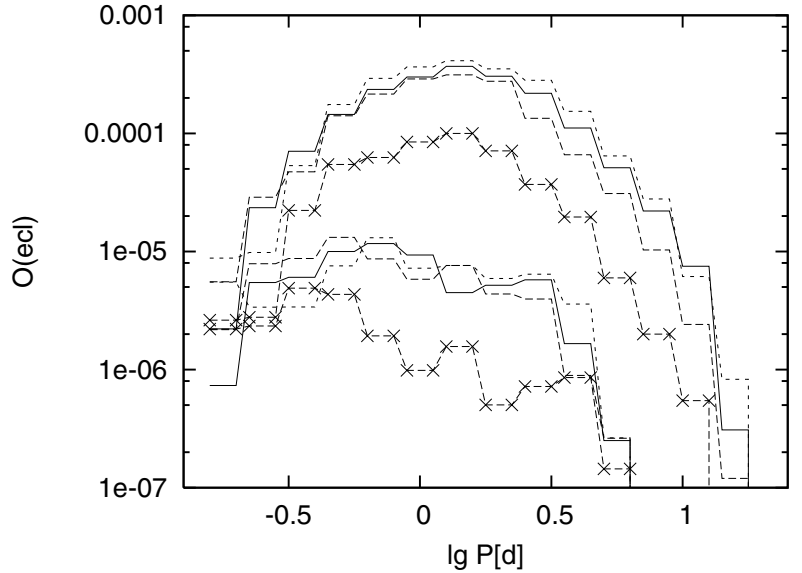

Fig. 9. Different versions of the $O_{\mathrm{e}}$-curves for $\Delta m>0.1$ and 0.8 , for the FG bin. The three closely spaced curves give results using the standard $q$-distribution (full line), the (DM) Q1 version (dashed), or the (uniform) Q2 version (dotted line). A factor 5 below the other curves (dashed line plus crosses) are the ones calculated with the random pairing Q3 input.

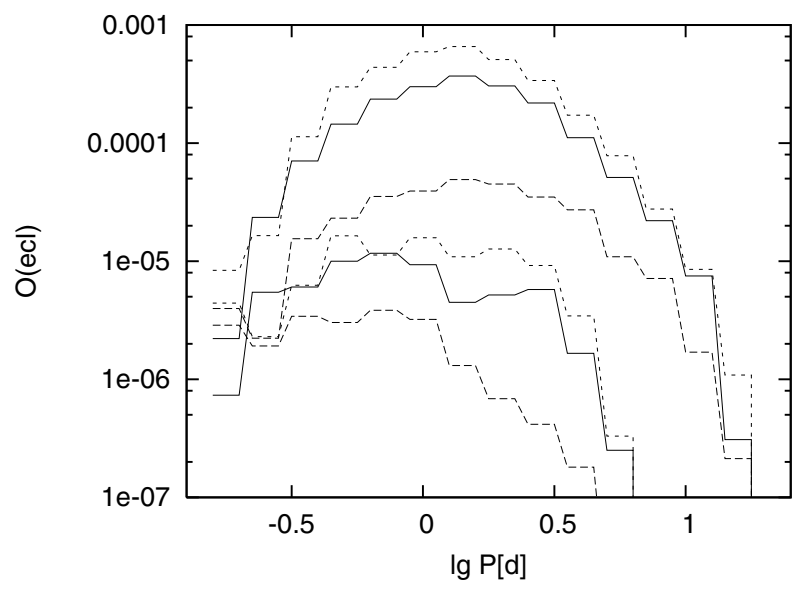

Fig. 10. Different versions of the $O_{\mathrm{e}}$-curves for $\Delta m>0.1$ and 0.8 , for the FG bin. The full lines are for the standard a-distribution, the dashed ones for the more peaked $\mathrm{A} 1$ version, and the dotted ones for the less peaked A2 version.

eclipsing binaries than the standard ones, clearly showing the problem faced by random pairing schemes in creating sufficient numbers of eclipsing systems.

Figure 10 shows the similar differential effects for the A1 and A2 modifications. Here, the A1 curve is much lower, and the A2 one clearly above the nominal. From similar calculations and plots along the main sequence, this seems part of a regular trend, with the largest effects at $\mathrm{L}$ and the smallest at O. Somewhat surprisingly, these $f(a)$-effects are close to a simplistic expectation looking only at the original $f(a)$-distributions. Figure 11 shows the raw number of systems for the three different $f(a)$-assumptions, and for three different mass-regions. To first order, the initial and final perioddistributions are proportional to each other, with the same evolutionary details preserved. The final $O_{\mathrm{e}}$-results do of course include also the changes in $P_{\mathrm{e}}$, actually turning the somewhat 


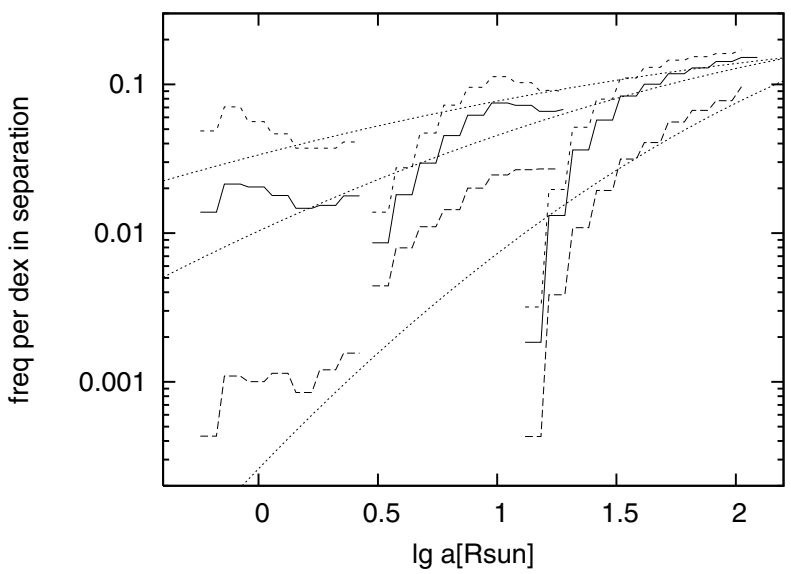

Fig. 11. The number of close binary systems as calculated with the standard $f(a)$ (full lines), with the A1 modification (long dashes) or with the A2 modification (short dashes). The dotted lines show the input distributions, and relevant parts of the data for the ML (left), FG (middle) and $\mathrm{O}$ (right) bins are shown.

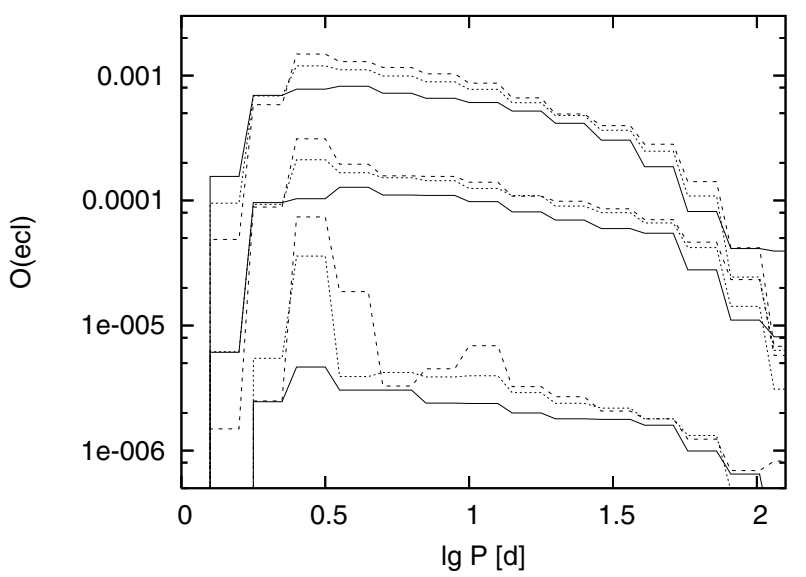

Fig. 12. The $O_{\mathrm{e}}$-curves for $\Delta m>0.1,0.4$ and 0.8 , for the $\mathrm{O}$ bin, for three different metallicities. The full curve is for a mean $[\mathrm{Fe} / \mathrm{H}]$ about -0.55 , the dashed line for +0.15 , and the dotted line for $[\mathrm{Fe} / \mathrm{H}]=-0.1$.

small A1-effect for the FG stars in Fig. 11 to the larger one in Fig. 10.

As for the effects of metallicity, they vary in a complex way along the main sequence. For many bins, we could compare two partial runs with $[\mathrm{Fe} / \mathrm{H}] \sim+0.15$ and $[\mathrm{Fe} / \mathrm{H}] \sim-0.55$, and generally, the $O_{\mathrm{e}}$-values are higher for the higher metallicity. In some simple cases, such as for the FG and $\mathrm{K}$ bins, the net effect is mainly a shift of $\lg O_{\mathrm{e}}$ in proportion to $[\mathrm{Fe} / \mathrm{H}]$, with a proportionality constant about 0.10 (per dex in metallicity) at FG or 0.20 at $\mathrm{K}$. For less massive stars, there is also a change-of-shape, with higher $O_{\mathrm{e}}$ for higher metallicity at the long-period edge, but equal of fewer systems at short periods. For the more massive stars, there is still a general rise, but then also a more pronounced effect on the Algol-type bump. This is both shifted towards longer periods, and increased in amplitude with a rise in metallicity. For O-stars, the general rise is of the order of 0.25 , but the probability for 3-day eclipsing binaries is very much dependent on $[\mathrm{Fe} / \mathrm{H}]$, as can be seen in Fig. 12 .

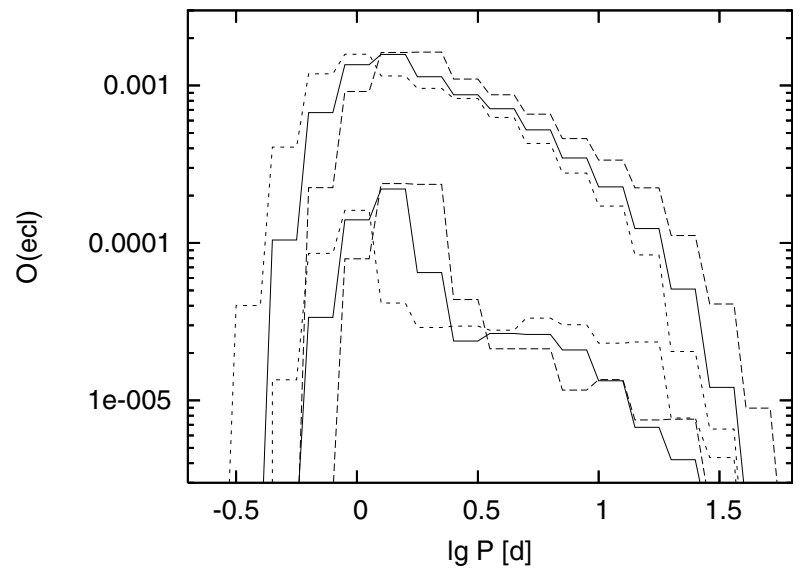

Fig. 13. The $O_{\mathrm{e}}$-curves for $\Delta m>0.1$ and 0.8 , for the B bin, for three different metallicities. The full curve is for a mean $[\mathrm{Fe} / \mathrm{H}]$ about -0.4 , the dashed line for +0.2 , and the dotted line for $[\mathrm{Fe} / \mathrm{H}]=-1.0$.

For the OB bin, the amplitude-effect is smaller, but we have an obvious period-shift by about 0.2 in $\lg P($ per dex in $[\mathrm{Fe} / \mathrm{H}])$.

At $\mathrm{B}$, the period-shift seems maximal, and in view of the interesting observational results for the LMC treated in Sect. 8, we made some extra runs with a larger range of metallicity. The BSE-program does not allow $z$-values above 0.032 , and the high- $z$ comparison is for a mean $[\mathrm{Fe} / \mathrm{H}]=0.21$. As a low- $z$ sample, we used a mean $[\mathrm{Fe} / \mathrm{H}]=-1.0$, and for an in-between set, a run with mean $[\mathrm{Fe} / \mathrm{H}]=-0.4$ was already available. Figure 13 shows again the $O_{\mathrm{e}}$-curves for $\Delta m>0.1$ and $\Delta m>0.8$. There seems to be a rather linear shift towards longer periods with higher metallicity, by about 0.25 in $\lg P$ per dex in metallicity. At A, the period-shift is down to about 0.1, and the amplitudeshift to only some 0.04 .

\section{Comparison with Hipparcos data}

There are few data sets where our theoretical curves can be compared with observations. A crude but reassuring check can however be obtained from the data in the Hipparcos Catalog (ESA 1997). The basic assumption is that most of the eclipsing binaries with periods in the 0.2 to 20 day range and eclipsedepths larger than $0.1 \mathrm{mag}$ were detected in the Hipparcos photometry. Apart from the eclipse detection issues (see below), the general completeness issues for Hipparcos are too complex to be taken rigorously into account. For the present crude check, we simply assumed a magnitude completeness limit $V=8.0$ at galactic latitudes lower than 30 degrees, and $V=9.0$ at higher latitudes. This bisection of the sky was chosen as a simplistic first step towards a more realistic sampling, but in order to get more than a few tens of eclipsing systems, we may have pushed the completeness below $50 \%$ at some latitudes (cf. Mignard 2000). As long as the incompleteness is not correlated with the fraction of eclipses, this should however not be a big problem.

Using the Celestia software (ESA 1998), we then simply made eclipse-statistics for two samples, one with parallaxes larger than 4.5 mas and the other with parallaxes in the 


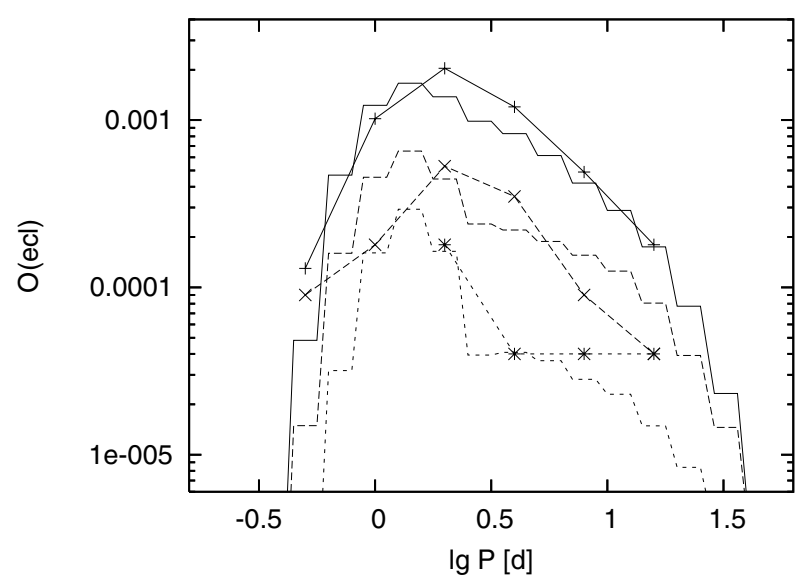

Fig. 14. The observed numbers of binaries in the distant Hipparcos sample, together with the theoretical (B) $O_{\mathrm{e}}$-curves. From above, the data are for $\Delta m>0.1,0.4$ and $0.7 \mathrm{mag}$.

interval 1.0 to 4.5 mas. On average, the more distant sample refers to brighter stars, and the aim of the division is to see some indication of the trend with absolute magnitude. The observed (small!) numbers were normalized by division by the total number of stars in the same range of $V$ and parallax (22 585 for the distant sample and 34335 for the closer one), giving observational ratios to be compared with our theoretical $O_{\mathrm{e}}$-data.

To make the comparison, we have to know the distribution of absolute magnitudes in the two samples. The $M_{V}$-distributions are quite wide $(10 / 90 \%$ percentiles at $-2.2 /+0.9$ for the distant one, $+0.3 / 4.0$ for the closer one), but they differ enough to influence appreciably the eclipse statistics. The median magnitudes are -0.35 and 1.98 , and comparing with Table 1, they are not too far from the theoretical medians for the $\mathrm{B}$ and the $\mathrm{A}$ bins. In view of the the many other uncertainties, no further refinement seems warranted, and Figs. 14 and 15 show the Hipparcos data together with the $O_{\mathrm{e}}$-curves. The agreement is good for the more luminous sample, while for the nearby sample, there seems to be more short-period pairs observed than predicted. This is smallnumber statistics (one system per bin at $\Delta m=0.7$ ), however, and the differences are not significant. The general agreement of the maximum $O_{\mathrm{e}}$-levels is however an indication both that our input $f(a)$ is reasonable and that the binary fraction is high (cf. Sect. 6).

Apart from a level-uncertainty due to the assumed binary fraction, our theoretical curves also use the standard discovery criterion with $\Delta m>0.1$ for $5 \%$ of the orbit. This is admittedly rather ad hoc, but it gives the reasonable large-period slope shown in Fig. 14. Requiring only 0.05 mag eclipses but for $10 \%$ of the orbit pushes the long-period part of the $O_{\mathrm{e}}$-curves down as compared with the observations, and $0.1 \mathrm{mag} / 10 \%$ even more so.

\section{Comparison with LMC data}

In recent years several surveys, e.g. MACHO, have been made looking for microlensing events to test the hypothesis that a significant fraction of the dark matter in the halo of the Milky Way

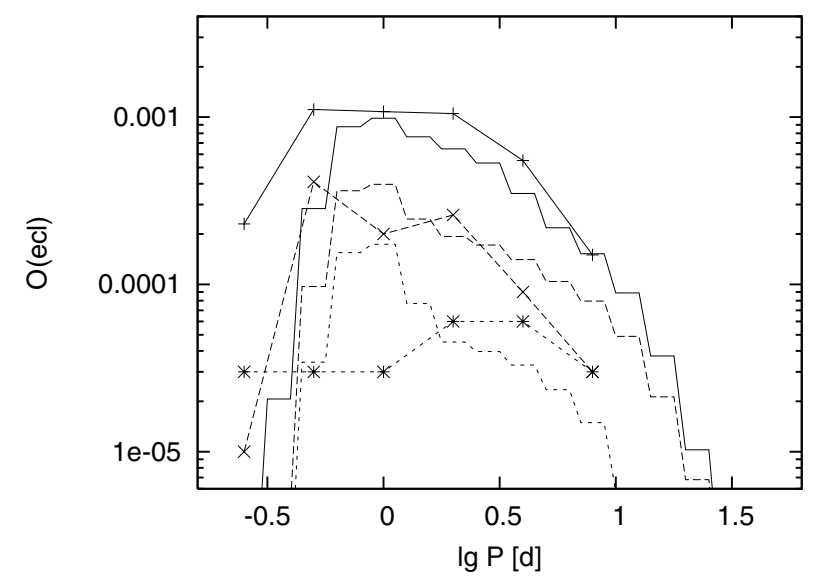

Fig. 15. The observed numbers of binaries in the close Hipparcos sample, together with the theoretical (A) $O_{\mathrm{e}}$-curves. From above, the data are for $\Delta m>0.1,0.4$ and $0.7 \mathrm{mag}$.

is made up of objects like brown dwarfs or planets. These surveys are a gold-mine for all studies of variable stars, e.g. eclipsing binaries. Several studies have been made from the data produced by MACHO, and in Alcock et al. (1997), the data from the study towards the LMC has been analyzed. They extracted there a sample of 611 eclipsing binaries by examining the light curves of all potential short-period variables. We have analyzed these data further regarding eclipse-depth and period, and we may thus compare our theoretical studies with this observed sample.

Looking at the color-magnitude plot in Alcock et al. (1997), the observations seem reasonably complete for apparent $V$ magnitudes brighter than 18.0. Using a distance modulus of 18.5 (Alves 2004), plus a schematic 0.5 mag absorption (cf. Zaritsky 1999), this corresponds to absolute magnitudes brighter than -1.0 , that is somewhere between our $\mathrm{OB}$ and $B$ bins. Because the shape of the theoretical curves do not vary fast with absolute magnitude, and in order to get a reasonably large sample, we made $P / \Delta m$ statistics for all systems with $V<$ $18, V-R<0.3$. (This color-cutoff should correspond roughly to our theoretical $V-I<0.5$, allowing for some slight reddening).

For the theoretical comparison, we first interpolate our $\mathrm{OB}$ and $\mathrm{B}$ results to an $[\mathrm{Fe} / \mathrm{H}]$ to around -0.34 (Luck et al. 1998) typical for young LMC-stars. We then use the mean absolute magnitude in our observed sample $(-2.2)$ plus the mean absolute magnitudes given in Table 1 to interpolate again to mean theoretical $O_{\mathrm{e}}$-curves.

Since the LMC data are not normalized, the observed histograms can be shifted arbitrarily in the y-direction, but from Fig. 16, we see at once that the observations put the maximum in the period-distribution for deep eclipses at a much longer period than expected from the theory. There is the predicted hump, but at the wrong period. A similar shift was found in Sect. 6, but the magnitude is much too high to be explained even by super-metal-rich stars. The large-scale variations in $f(a)$ and $f(q)$ as explored in Sect. 6 also do not give a shift of the period-maximum, and we have to make sure that we do not overlook any possible systematic effects in the observations. 


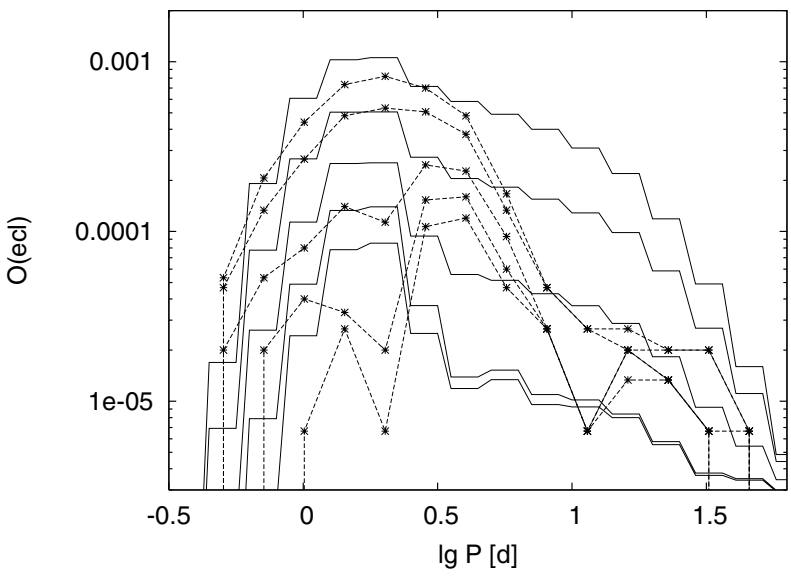

Fig. 16. The distribution of eclipse depth as function of period for OB-stars in the LMC. The solid curves are our theoretical $O_{\text {e pre- }}$ dictions for $\Delta m=0.2,0.4,0.6,0.8$, and 1.0. The dashed lines are the observed MACHO data (arbitrarily shifted in the $y$ direction).

A first necessary test is of course to try to see any changes in the observed curves with respect to absolute(=apparent) magnitude, noting the theoretical prediction of longer hump periods for brighter stars. No such effects could be seen for different magnitude and color cutoffs (and only 5 out of 563 systems are bright enough to be in our $\mathrm{O}$ bin). It is also clear that many of these close binaries show large out-of-eclipse light-variations. Because we use the full max-min amplitude, we overestimate the eclipse-depths, and more so the shorter the period. This will in effect aggravate the fit to the theory, depressing the observed curves at short periods which are already too low. Similarly, the detection completeness should be best at short periods, again going against the observed effect. The only slight correction from longer to shorter periods would be where a short-period system with small secondary minimum is miss-classified as a double-period system with equal minima. Such cases may occur, but not in enough numbers to make a qualitative change.

Although it seems very plausible that some of the internal BSE parameters governing close binary evolution need adjustment, it is hard to see why these adjustments should be so different between LMC and the Galaxy. The reason for the bad fit in Fig. 16 is thus unclear, but some small-scale peculiarities in the birth $f(a)$-distribution and/or the interstellar environment (influencing the mass-loss/mass-transfer processes) may perhaps be involved.

\section{Conclusions and directions for future studies}

There are a number of conclusions to be drawn from the present investigation. The most obvious one is of course that for predictions about eclipsing binaries, one has to allow for the full complexity of close binary evolution, and that only with the availability of a rapid interpolation tool like BSE, are multimillion system population syntheses at all feasible. In this first study, we have used BSE with standard parameters, but for increased realism, some of them should be optimized further (see below).
Our main result is a theoretical prediction about the periods and depths of main sequence eclipsing binaries, as a function of their absolute magnitude. An admittedly crude comparison with real Hipparcos observations shows a quite reasonable fit for the number of eclipsing binaries, and even for their distribution over period and eclipse depth. Encouraged by this fit, we have tried also to estimate the properties of the eclipsing binaries observable by ESA's Gaia mission, see Dischler \& Söderhjelm (2005) and Söderhjelm (2005). For B-stars in the LMC, we could only look at the $P / \Delta m$ distributions, which is however sufficiently offset from the theoretical one that one is led to assume some rather different input orbit-distributions than in the Galaxy.

As a general result, we have concluded that the number of observed eclipsing binaries scales rather directly with the input frequency of short-period orbits, although the post-evolution period-distribution is far from the input one. The good fit for the Hipparcos samples shows that at least in the short-period end, the simple log-normal Duquennoy-Mayor a-distribution (as derived originally for G-stars) is a good model even for the upper main sequence. As for the mass-ratios, the present results (not unexpectedly) rule out all $q$-distributions with an excess of small-mass secondaries, as obtained e.g. by choosing the primary and secondary masses from the same IMF. For flatter distributions, small details (like the $q=1$ peak) turn out to have rather little influence on the observable eclipsing-binary population.

Already from our existing data, we may go on to derive theoretical distributions of spectroscopic and visual binaries in the same main-sequence result-bins. Comparisons with observed data will then allow conclusions about $f(a)$ over a much larger a-interval. To improve the results for the eclipsing binaries, the obvious next step is to calculate more realistic light-curves, with outside-eclipse variations and limb-darkening. Also, with enough computer resources, it should be interesting to perform large-scale studies where also some of the internal BSE parameters are varied, and the results should be given for smaller areas of the HR-diagram.

Acknowledgements. We thank Jarrod Hurley for giving us free access to the crucial BSE-code, Chris Tout for some advice as to how to use it, and Onno Pols for a thorough refereeing of the final article.

\section{Appendix A: Sample eclipse probabilities}

Below are given two sample $P_{\mathrm{e}} / Q_{\mathrm{e}}$ tables, giving the data for $\Delta m=0.1$ and 0.8 at 18 different periods (spacing 0.15 in $\lg P$ ). Each row corresponds to a MS bin (with brown dwarfs in the $\mathrm{ML}+\mathrm{L}$ combination). The column headings give the mean period in days, and in Table A.1 are given the corresponding mean $\lg P_{\mathrm{e}}$ values. In Table A.2, we give instead $\lg O_{\mathrm{e}}$, where $O_{\mathrm{e}}$ is the fraction of eclipsing binaries (per 0.3 dex in period) relative to all stars in this bin, assuming $80 \%$ binarity in our population synthesis model. 
Table A.1. $P_{\mathrm{e}}$-values.

\begin{tabular}{|c|c|c|c|c|c|c|c|c|c|c|c|c|c|c|c|c|c|c|}
\hline & Z & 1 & & & & & & & & & & & & & & & & \\
\hline Bin & 0.175 & 0.25 & 0.36 & 0.51 & 0.72 & 1.02 & 1.44 & 2.04 & 2.88 & 4.08 & 5.76 & 8.16 & 11.5 & 16.3 & 23.0 & 32.5 & 46 & 65 \\
\hline $\mathrm{O}$ & - & - & - & - & - & - & -0.50 & -0.39 & -0.55 & -0.79 & -1.00 & -1.13 & -1.25 & -1.39 & -1.54 & -1.71 & -1.91 & -2.30 \\
\hline OB & - & - & - & -0.22 & -0.31 & -0.36 & -0.44 & -0.55 & -0.74 & -0.92 & -1.04 & -1.21 & -1.41 & -1.61 & -1.89 & -2.29 & -2.74 & -3.31 \\
\hline B & -0.87 & -1.00 & -0.74 & -0.43 & -0.42 & -0.49 & -0.63 & -0.83 & -1.05 & -1.16 & -1.32 & -1.53 & -1.74 & -2.00 & -2.39 & -2.94 & -3.76 & -4.30 \\
\hline A & -0.92 & -0.95 & -0.79 & -0.68 & -0.69 & -0.84 & -1.04 & -1.17 & -1.26 & -1.46 & -1.69 & -1.89 & -2.16 & -2.59 & -3.18 & -4.13 & -5.61 & - \\
\hline FG & -1.05 & -0.89 & -0.87 & -0.95 & -1.05 & -1.18 & -1.29 & -1.49 & -1.75 & -1.98 & -2.25 & -2.70 & -3.32 & -4.30 & -5.92 & - & - & - \\
\hline K & -0.93 & -0.91 & -0.95 & -1.03 & -1.31 & -1.63 & -1.77 & -1.92 & -2.17 & -2.59 & -3.57 & - & - & - & - & - & - & - \\
\hline $\mathrm{M}(\mathrm{a})$ & -0.70 & -0.86 & -1.15 & -1.37 & -1.44 & -1.60 & -1.81 & -2.16 & -2.92 & -4.48 & - & - & - & - & - & - & - & - \\
\hline $\mathrm{M}(\mathrm{b})$ & -0.78 & -1.16 & -1.61 & -1.73 & -1.86 & -2.16 & -2.58 & -3.64 & - & - & - & - & - & - & - & - & - & - \\
\hline \multirow[t]{2}{*}{$\mathrm{ML}+\mathrm{L}$} & -1.00 & -1.45 & -1.80 & -2.15 & -2.56 & -3.31 & -4.35 & -6.41 & - & - & - & - & - & - & - & - & - & - \\
\hline & \multicolumn{18}{|c|}{$\Delta M \geq 0.8$} \\
\hline $\mathrm{O}$ & - & - & - & - & - & - & - & -3.32 & -2.28 & -3.64 & - & - & -4.21 & - & - & -5.55 & -5.49 & -5.15 \\
\hline OB & - & - & - & - & - & -2.42 & -1.77 & -1.59 & -1.97 & -3.26 & -3.09 & -3.28 & -3.26 & -3.23 & -3.46 & -3.43 & -3.43 & -3.62 \\
\hline B & -1.35 & -2.21 & -1.80 & -2.55 & -1.81 & -1.51 & -1.47 & -1.84 & -2.58 & -2.57 & -2.65 & -2.81 & -2.95 & -3.17 & -3.42 & -3.81 & -4.46 & -4.39 \\
\hline A & -1.42 & -1.52 & -1.96 & -1.81 & -1.58 & -1.70 & -2.19 & -2.53 & -2.59 & -2.70 & -2.91 & -3.23 & -3.94 & -5.52 & -6.33 & -6.58 & -6.76 & - \\
\hline FG & -1.23 & -1.45 & -1.87 & -2.10 & -2.39 & -2.83 & -3.01 & -3.22 & -3.45 & -3.69 & -4.45 & - & - & - & - & - & - & - \\
\hline K & -1.22 & -1.53 & -2.11 & -2.26 & -2.76 & -3.06 & -3.18 & -3.14 & -3.21 & -3.51 & -4.45 & - & - & - & - & - & - & - \\
\hline $\mathrm{M}(\mathrm{a})$ & -2.65 & -3.09 & -3.68 & -4.44 & -4.76 & -5.04 & -5.10 & -5.57 & - & - & - & - & - & - & - & - & - & - \\
\hline $\mathrm{M}(\mathrm{b})$ & -2.36 & -2.59 & -2.96 & -3.24 & -3.40 & -3.71 & -4.40 & -5.21 & - & - & - & - & - & - & - & - & - & - \\
\hline $\mathrm{ML}+\mathrm{L}$ & -1.38 & -1.77 & -2.15 & -2.57 & -3.08 & -3.92 & -4.86 & -7.39 & - & - & - & - & - & - & - & - & - & - \\
\hline
\end{tabular}

Table A.2. $O_{\mathrm{e}}$-values.

\begin{tabular}{|c|c|c|c|c|c|c|c|c|c|c|c|c|c|c|c|c|c|c|}
\hline \multirow[b]{2}{*}{ Bin } & \multicolumn{18}{|c|}{$\Delta M \geq 0.1$} \\
\hline & 0.175 & 0.25 & 0.36 & 0.51 & 0.72 & 1.02 & 1.44 & 2.04 & 2.88 & 4.08 & 5.76 & 8.16 & 11.5 & 16.3 & 23.0 & 32.5 & 46 & 65 \\
\hline $\mathrm{O}$ & - & - & - & - & - & - & -4.00 & -3.17 & -2.93 & -2.96 & -3.01 & -3.06 & -3.12 & -3.22 & -3.33 & -3.45 & -3.62 & -3.97 \\
\hline OB & - & - & - & -5.95 & -4.16 & -3.29 & -2.91 & -2.77 & -2.87 & -3.00 & -3.08 & -3.21 & -3.36 & -3.50 & -3.72 & -4.05 & -4.49 & -5.04 \\
\hline B & -6.59 & -6.87 & -5.97 & -4.32 & -3.33 & -2.91 & -2.78 & -2.86 & -3.01 & -3.08 & -3.21 & -3.38 & -3.54 & -3.76 & -4.11 & -4.63 & -5.42 & -5.94 \\
\hline A & -5.82 & -5.87 & -4.69 & -3.55 & -3.06 & -3.01 & -3.12 & -3.19 & -3.27 & -3.46 & -3.66 & -3.82 & -4.05 & -4.43 & -4.99 & -5.92 & -7.37 & - \\
\hline FG & -5.33 & -4.78 & -4.24 & -3.87 & -3.67 & -3.57 & -3.51 & -3.57 & -3.74 & -3.98 & -4.30 & -4.75 & -5.32 & -6.26 & -7.84 & - & - & - \\
\hline K & -5.18 & -4.83 & -4.51 & -4.20 & -4.13 & -4.11 & -4.02 & -4.05 & -4.31 & -4.74 & -5.70 & - & - & - & - & - & - & - \\
\hline $\mathrm{M}(\mathrm{a})$ & -3.94 & -3.86 & -3.93 & -3.98 & -3.93 & -4.08 & -4.24 & -4.52 & -5.22 & -6.71 & - & - & - & - & - & - & - & - \\
\hline $\mathrm{M}(\mathrm{b})$ & -3.65 & -3.80 & -4.09 & -4.18 & -4.38 & -4.70 & -5.05 & -6.07 & - & - & - & - & - & - & - & - & - & - \\
\hline \multirow[t]{2}{*}{$\mathrm{ML}+\mathrm{L}$} & t.09 & -4.20 & -4.37 & -4.67 & -5.17 & -5.94 & -6.92 & -8.91 & - & - & - & - & - & - & - & - & - & - \\
\hline & \multicolumn{18}{|c|}{$\Delta M \geq 0.8$} \\
\hline $\mathrm{O}$ & - & - & - & - & - & - & - & -6.10 & -4.66 & -5.81 & - & - & -6.08 & - & - & -7.29 & -7.20 & -6.81 \\
\hline OB & - & - & - & - & - & -5.35 & -4.23 & -3.81 & -4.10 & -5.35 & -5.13 & -5.27 & -5.21 & -5.12 & -5.29 & -5.19 & -5.18 & -5.34 \\
\hline B & -7.08 & -8.07 & -7.03 & -6.44 & -4.72 & -3.93 & -3.62 & -3.86 & -4.55 & -4.49 & -4.54 & -4.65 & -4.75 & -4.93 & -5.15 & -5.50 & -6.12 & -6.04 \\
\hline A & -6.32 & -6.44 & -5.86 & -4.67 & -3.95 & -3.87 & -4.26 & -4.55 & -4.61 & -4.70 & -4.87 & -5.15 & -5.83 & -7.36 & -8.14 & -8.36 & -8.52 & - \\
\hline FG & -5.52 & -5.34 & -5.24 & -5.02 & -5.00 & -5.22 & -5.23 & -5.30 & -5.44 & -5.70 & -6.50 & - & - & - & - & - & - & - \\
\hline $\mathrm{K}$ & -5.47 & -5.46 & -5.66 & -5.43 & -5.58 & -5.55 & -5.43 & -5.27 & -5.36 & -5.66 & -6.58 & - & - & - & - & - & - & - \\
\hline $\mathrm{M}(\mathrm{a})$ & -5.89 & -6.09 & -6.47 & -7.04 & -7.24 & -7.52 & -7.53 & -7.93 & - & - & - & - & - & - & - & - & - & - \\
\hline $\mathrm{M}(\mathrm{b})$ & -5.24 & -5.24 & -5.43 & -5.69 & -5.92 & -6.25 & -6.87 & -7.64 & - & - & - & - & - & - & - & - & - & - \\
\hline $\mathrm{ML}+\mathrm{L}$ & -4.47 & -4.53 & -4.73 & -5.09 & -5.69 & -6.55 & -7.43 & -9.89 & - & - & - & - & - & - & - & - & - & - \\
\hline
\end{tabular}




\section{References}

Alcock, C., Allsman, R. A., Alves, D., et al. 1997, AJ, 114, 326

Alves, D. R. 2004, New Astron. Rev., 48, 659

Antonello, E., Farinella, P., Guerrero, G., Mantegazza, L., \& Paolicchi, P. 1980, Ap\&SS, 72, 359

Baraffe, I., Chabrier, G., Allard, F., \& Hauschildt, P. H. 1998, A\&A, 337,403

Dischler, J., \& Söderhjelm, S. 2005, in Proc. The three-dimensional Universe with Gaia, ed. C. Turon, K. S. O'Flaherty, \& M. A. C. Perryman, ESA SP-576, 569

Duquennoy, A., \& Mayor, M. 1991, A\&A, 248, 485

ESA 1997, The Hipparcos and Tycho Catalogues, ESA SP-1200

ESA 1998, Celestia 2000, ESA SP-1220

Farinella, P., \& Paolicchi, P. 1978, Ap\&SS, 54, 389

Flower, P. J. 1996, ApJ, 469, 355

Halbwachs, J. L., Mayor, M., Udry, S., \& Arenou, F. 2003, A\&A, 397, 159
Hurley, J. R., Pols, O. R., \& Tout, C. A. 2000, MNRAS, 315, 543

Hurley, J. R., Tout, C. A., \& Pols, O. R. 2002, MNRAS, 329, 897

Irwin, J. B. 1962, in Stars and Stellar Systems, Astronomical Techniques, ed. W. A. Hiltner (Univ. Chicago Press), 2, 584

Kroupa, P. 2001, MNRAS, 322, 231

Luck, R. R., Moffett, T. J., Barnes III, T. G., \& Gieren, W. P. 1998, AJ, 115,605

Malkov, O., \& Zinnecker, H. 2001, MNRAS, 321, 149

Mazeh, T., Simon, M., Prato, L., Markus, B., \& Zucker, S. 2003, ApJ, 599,1344

Mignard, F. 2000, A\&A, 354, 522

Nordström, B., Mayor, M., Andersen, J., et al. 2004, A\&A, 418, 989

Söderhjelm, S. 2000, AN, 321, 165

Söderhjelm, S. 2005, in Proc. The three-dimensional Universe with Gaia, ed. C. Turon, K. S. O'Flaherty, \& M. A. C. Perryman, ESA SP-576, 97

Zaritsky, D. 1999, AJ, 118, 2824 